\title{
FIGO Stage IIIC Ovarian Cancer
}

National Cancer Institute

\section{Source}

National Cancer Institute. FIGO Stage IIIC Ovarian Cancer. NCI Thesaurus. Code

C128093.

Ovarian cancer with macroscopic, extrapelvic, peritoneal metastasis greater than $2 \mathrm{~cm}$ +/- positive retroperitoneal lymph nodes. Includes extension to capsule of liver/spleen. (FIGO, 2014) 